\title{
Is there a future for xenon anesthesia?
}

Takahisa Goto MD

$\mathrm{X}$

ENON is an inert gas that is well known as a material for lamps. Although named after a Greek word "stranger", xenon is becoming less and less of a stranger for us anesthesiologists. In this regard, it is timely that this month's issue of the Journal publishes an article by Nakayama, et al. on the cardiac effect of xenon. ${ }^{1}$ The authors demonstrated that neither xenon nor nitrous oxide $\left(\mathrm{N}_{2} \mathrm{O}\right) 50 \%$ affected the contractile state of the Langendorff's isolated and perfused heart when compared with nitrogen $\mathbf{5 0 \%}$.

\section{Historical perspective}

It has long been recognized that xenon is an anesthetic. Its minimum alveolar concentration is $71 \%{ }^{2}$ or maybe slightly lower, ${ }^{3}$ indicating that it is more potent than $\mathrm{N}_{2} \mathrm{O}$. However, the extremely high cost (approximately $\$ 10.00$ US per litre, which is 100 times more than the cost of $\mathrm{N}_{2} \mathrm{O}$ ) has hindered its wide clinical application. In fact, xenon was completely forgotten, for more than 30 years since the early clinical trials in the 1950's, ${ }^{4,5}$ until Drs. Lachmann, Erdmann and their colleagues at Rotterdam rediscovered it in 1990.6 Since then, there has been a growing interest in xenon, especially in Europe and Japan, and two multicenter clinical trials have been completed in the European Union. In Russia, xenon is approved for clinical use. However, cost remains a significant problem, as it was $\mathbf{5 0}$ years ago. Therefore, one may naturally wonder why xenon is attracting interest at this time of cost containment. I think there are three major reasons.

\section{Clinical advantages of xenon}

First, xenon has many of the properties of an ideal anesthetic. For example, its blood/gas partition coefficient $(0.12)^{7}$ is lower than that of any other anesthetic, and this enables rapid induction of and emergence from anesthesia. ${ }^{8,9}$ Therefore, it was originally thought that xenon would be ideal for ambulatory anesthesia. However, this is probably unrealistic because xenon often causes nausea and vomiting. ${ }^{9,10}$
Recently, investigators have started to recognize another very appealing characteristic of xenon, i.e., its lack of cardiac depression. The study of Nakayama et al. in this issue of the Journal ${ }^{1}$ is important because it convincingly supports this. The results are consistent with xenon's lack of action on some important cardiac ion channels, ${ }^{11,12}$ and also with its lack of hemodynamic depression in healthy humans, ${ }^{13}$ in patients with ischemic heart disease, ${ }^{14}$ and in dogs with normal hearts and with cardiomyopathy. ${ }^{15}$ Furthermore, xenon produces analgesia ${ }^{10,16}$ and thereby suppresses hemodynamic and catecholamine responses to surgical stimulation, ${ }^{17,18}$ further contributing to hemodynamic stability. In addition, unlike other analgesics, xenon is a potent hypnotic. ${ }^{19,20}$ The unique combination of analgesia, hypnosis, and lack of hemodynamic depression in one agent would make xenon a very attractive choice for patients with limited cardiovascular reserve and may compensate for its high cost. This prediction is worth being tested by sufficiently large clinical trials.

\section{Xenon as a scientific tool}

Second, xenon is an interesting scientific tool with which to investigate the mechanism(s) of anesthesia. Although it has been shown that xenon inhibits the function of the $\mathrm{N}$-methyl-D-aspartate subtype of the glutamate recep$\operatorname{tor}^{21,22}$ and also of the nicotinic acetylcholine receptor, ${ }^{22}$ how it does so remains a mystery. Because xenon is inert and, therefore, displays an extremely low chemical reactivity, it may alter the function of these receptors via physicochemical means, or these receptors may not be as important as we would like to believe. Another provocative finding is that the hypnotic states produced by xenon and volatile anesthetic are electrophysiologically similar $^{20}$ despite the fact that xenon hardly affects the function of the gamma-aminobutyric acid $_{\mathrm{A}}$ receptor, ${ }^{21,22}$ that putatively mediates the action of many anesthetics.

\section{Xenon and the global environment}

The third reason includes the environmental friendliness of xenon, which strongly appeals to the increasing

From the Department of Anesthesia, Teikyo University Ichihara Hospital, Ichihara-shi, Japan.

Address correspondence to: Dr. Takahisa Goto, Department of Anesthesia, Teikyo University Ichihara Hospital, 3426-3 Anesaki, Ichiharashi, Chiba-ken 299-0111, Japan. Phone: +81-436-62-1211, ext. 2716; Fax: +81-436-62-2621; E-mail: takigoto@med.teikyo-u.ac.jp 
number of ecologically minded people in the anesthesia community (myself included). Xenon is produced by fractional distillation of liquefied air (as a by-product of oxygen and nitrogen). Therefore, it does not add to atmospheric pollution when emitted from the anesthesia circuit because it simply goes back to the atmosphere. In contrast, all the inhalational anesthetics we currently use pollute the atmosphere when released from the anesthesia system because they are synthesized artificially. This is a significant issue. For example, isoflurane is a hydrogenated chlorofluorocarbon possessing ozone-depleting potential. It was internationally agreed upon that manufacture of many compounds of this class be totally banned by the year 2030 unless exempt. ${ }^{23}$ $\mathrm{N}_{2} \mathrm{O}$ is 230 times more potent as a greenhouse gas than is carbon dioxide on a molecular basis, and $\mathrm{N}_{2} \mathrm{O}$ released as a waste anesthetic contributes roughly $0.1 \%$ of the whole global warming. ${ }^{24}$ Because anesthesiologists constitute only $0.006 \%$ of the whole national population in the case of Japan, it is estimated that one anesthesiologist causes 15 times $(0.1 / 0.006)$ more global warming than do other people. To make things worse, the lifetime of $\mathrm{N}_{2} \mathrm{O}$ in the atmosphere is long approximately - 120 years. $^{24}$

\section{Concluding remarks}

There is a growing concern that mankind may not be able to live through the $2 \mathrm{l}^{\text {st }}$ century if the global warming and other forms of atmospheric pollution continue at the present rate. It is clear that we cannot continue the mass production, mass consumption, and mass wastage of the $20^{\text {th }}$ century, although this was exactly how many of us practiced (and, unfortunately, are still practicing) anesthesia. In the near future, the use of very low fresh gas flows or closed breathing systems, and even recycling of waste anesthetics, may become routine parts of our practice. When this happens, clinical application of xenon will be more feasible because the limited supply and high cost will be less of an impediment. What we should do at present is not to discard xenon simply because of its cost or to blindly welcome it just because it looks like an ideal anesthetic. Instead, we should keep accumulating knowledge on this noble gas so that we can solidly analyze its costbenefit balance. Such analyses should take into consideration not only patient care but also care of the planet earth, i.e., the global environment.

\section{Le xénon a-t-il un avenir en anesthésie ?}

Le xénon est un gaz inerte bien connu comme matériel d'éclairage. Ainsi appelé d'après un mot grec signifiant "étranger", le xénon est devenu de moins en moins étranger pour les anesthésiologistes que nous sommes. À ce propos, la publication dans le présent numéro du Journal de l'article de Nakayama, et coll. sur l'effet cardiaque du xénon survient à point nommé. ${ }^{1}$ Les auteurs y démontrent que ni le xénon, ni le protoxyde d'azote $\left(\mathrm{N}_{2} \mathrm{O}\right)$ à $50 \%$, comparés à l'azote à $50 \%$, n'ont d'effet sur la contractilité des cœurs de rats Langendorff isolés et perfusés.

\section{Historique}

Les qualités anesthésiques du xénon sont connues depuis longtemps. Sa concentration alvéolaire minimale de $71 \%^{2}$ ou peut-être un peu plus faible, ${ }^{3}$ indique qu'il est plus puissant que le $\mathrm{N}_{2} \mathrm{O}$. Son coût extrêmement élevé (environ 10,00 \$ US le litre, donc 100 fois plus cher que le $\mathrm{N}_{2} \mathrm{O}$ ) a cependant nui à son application clinique étendue. De fait, le xénon a été complètement oublié pendant plus de 30 ans depuis les premiers essais cliniques des années $1950^{4,5}$ jusqu'à ce que les Drs Lachmann, Erdmann et leurs collègues de Rotterdam le redécouvrent en 1990.6 Depuis, on s'y intéresse de plus en plus, surtout en Europe et au Japon, et deux essais cliniques multicentriques ont été réalisés au sein de l'Union européenne. En Russie, il est approuvé pour usage clinique. Mais, son coût élevé demeure un important problème. Pourquoi alors suscite-t-il autant d'intérêt en cette période de compression budgétaire ? Trois raisons principales nous semblent l'expliquer.

\section{Les avantages cliniques $d u$ xénon}

La première raison, c'est que le xénon a de nombreuses propriétés d'un anesthésique idéal. Ainsi, son coefficient de répartition sang/gaz $(0,12)^{7}$ est plus bas que celui de tout autre anesthésique, ce qui permet un rapide retour à la conscience après l'anesthésie. ${ }^{8,9} \mathrm{Il}$ a donc été considéré d'abord comme idéal pour l'anesthésie ambulatoire. Ce qui n'est sans doute pas réaliste, puisqu'il cause souvent des nausées et des vomissements. ${ }^{9,10}$

Récemment, des chercheurs ont commencé à entrevoir une autre caractéristique vraiment intéressante du xénon, il n'entraine pas de dépression cardiaque. De ce point de vue, l'étude de Nakayama et 
coll. publiée dans le présent numéro ${ }^{1}$ est importante, car elle vient appuyer cette affirmation de façon convaincante. Les résultats cadrent avec l'absence d'action du xénon sur d'importants canaux ioniques cardiaques, ${ }^{11,12}$ et aussi avec l'absence de dépression hémodynamique chez des sujets en bonne santé ${ }^{13}$ ou qui présentent une cardiopathie ischémique ${ }^{14}$ et chez des chiens dont le cour est normal ou qui sont atteints de cardiomyopathie. ${ }^{15}$ De plus, le xénon produit de l'analgésie ${ }^{10,16}$ et, donc, supprime les réponses hémodynamiques et de la catécholamine à la stimulation chirurgicale, ${ }^{17,18}$ contribuant davantage à la stabilité hémodynamique. De plus, contrairement à d'autres analgésiques, le xénon est un puissant hypnotique. ${ }^{19,20}$ La combinaison unique de ces effets analgésique, hypnotique et sans effet dépresseur myocardique en un seul agent en fait un choix très attrayant pour les patients dont la réserve cardio-vasculaire est limitée. Ces caractéristiques pourraient aussi compenser son coût élevé. Cette prédiction vaut la peine d'être vérifiée par des essais cliniques comportant un échantillonnage suffisant.

\section{Le xénon en qualité d'outil scientifique}

La seconde raison de l'attrait du xénon c'est qu'il est un outil scientifique digne de mention avec lequel on peut explorer les mécanismes de l'anesthésie. Il a été démontré que le xénon inhibe la fonction d'un soustype de récepteur glutamate, le $\mathrm{N}$-méthyl-Daspartate $^{21,22}$ et aussi celle du récepteur de l'acétylcholine nicotinique, ${ }^{22}$ mais on ne sait toujours pas comment il produit l'inhibition. Comme le xénon est un gaz inerte et, de ce fait, présente une réactivité chimique extrêmement faible, il pourrait modifier la fonction de ces récepteurs par des moyens physicochimiques, ou ces récepteurs seraient moins importants que nous avons voulu le croire. Autre résultat attrayant, l'état hypnotique produit par le xénon et un anesthésique volatil sont électrophysiologiquement semblables ${ }^{20}$ malgré le fait que le xénon modifie à peine la fonction du récepteur de l'acide gammaaminobutyrique, ${ }^{21,22}$ lequel pourrait agir comme médiateur de l'action de nombreux anesthésiques.

\section{Le xénon et l'environnement mondial}

La troisième raison concerne le respect de l'environnement que permet l'usage du xénon, ce qui lance un appel pressant à un nombre croissant d'anesthésiologistes, dont moi-même, préoccupés d'écologie. Le xénon est produit par la distillation fractionnée d'air liquéfié (donc un sous-produit de la concentration d'oxygène et d'azote). Pour cette raison, sa présence ne contribue pas à la pollution de l'air lorsqu'il est éliminé du circuit d'anesthésie, puisqu'il retourne tout simplement dans l'atmosphère. Par ailleurs, tous les anesthésiques d'inhalation que nous utilisons couramment polluent lorsqu'ils sont libérés du système d'anesthésie, car ils sont synthétisés artificiellement. C'est une question d'importance. Ainsi, l'isoflurane est un chlorofluorocarbure hydrogéné possédant un potentiel d'appauvrissement de la couche d'ozone. L'abolition totale pour l'année 2030, à moins d'exemption, de la préparation de nombreux composés de cette catégorie a été approuvée internationalement. ${ }^{23} \mathrm{Le} \mathrm{N}_{2} \mathrm{O}$ est 230 fois plus puissant comme gaz à effet de serre que le gaz carbonique, au plan moléculaire, et le $\mathrm{N}_{2} \mathrm{O}$ rejeté comme anesthésique expiré contribue à environ $0,1 \%$ du réchauffement du globe. ${ }^{24}$ Les anesthésiologistes formant seulement $0,006 \%$ de la population totale du Japon, on peut présumer qu'un anesthésiologiste cause 15 fois $(0,1 / 0,006)$ plus de réchauffement à l'échelle planétaire que ne le font les autres personnes. Pire encore, la durée de vie du $\mathrm{N}_{2} \mathrm{O}$ dans l'atmosphère est longue, approximativement 120 ans. $^{24}$

\section{Conclusion}

On s'inquiète de plus en plus à savoir si le monde peut survivre au $2 \mathrm{I}^{\mathrm{C}}$ siècle si le réchauffement de la planète et d'autres formes de pollution atmosphérique continuent au rythme actuel. C'est certain, nous ne pouvons poursuivre la production, la consommation et le gaspillage de masse du $20^{\mathrm{e}}$ siècle, même si c'est exactement ainsi que nombre d'entre nous avons pratiqué et, malheureusement, pratiquons encore l'anesthésie. Dans un proche avenir, l'utilisation de très bas débits de gaz frais ou de systèmes à circuits fermés et même la récupération des gaz expirés pourraient être de pratique courante. Quand cette situation aura cours, l'application clinique du xénon sera plus facilement réalisable parce que l'approvisionnement limité et le coût élevé seront considérés comme de moindres obstacles. Présentement, il ne faut pas écarter le xénon sous le seul prétexte de son coût ou l'utiliser aveuglément parce qu'il apparaît comme l'anesthésique idéal. Il faut plutôt chercher à mieux connaitre ce gaz noble de façon à pouvoir en faire une solide étude de rendement. Des analyses semblables devront tenir compte non seulement du patient, mais aussi de la planète terre, ce qui veut dire de l'environnement mondial.

\section{References}

1 Nakayama H, Takabashi H, Okubo N, Miyabe M, Toyooka $H$. Effects of xenon on cardiac function in isolated rat hearts under mild hypoxia: comparison with nitrous oxide. Can J Anesth 2002; 49: 375-79. 
2 Cullen SC, Eger EI II, Cullen BF, Gregory P. Observations on the anesthetic effect of the combination of xenon and halothane. Anesthesiology 1969; 31: 305-9.

3 Nakata $\Upsilon$, Goto T, Ishiguro $\Upsilon$, et al. Minimum alveolar concentration (MAC) of xenon with sevoflurane in humans. Anesthesiology 2001; 94: 611-4.

4 Cullen SC, Gross EG. The anesthetic properties of xenon in animals and human beings, with additional observations on krypton. Science 1951; 113: 580-2.

5 Morris LE, Knott JR, Pittinger CB. Electro-encephalographic and blood gas observations in human surgical patients during xenon anesthesia. Anesthesiology 1955; 16: 312-9.

6 Lachmann B, Armbruster S, Schairer W, et al. Safety and efficacy of xenon in routine use as an inhalational anaesthetic. Lancet 1990; 335: 1413-5.

7 Goto T, Suwa K, Uezono S, Ichinose F, Uchiyama M, Morita $S$. The blood-gas partition coefficient of xenon may be lower than generally accepted. Br J Anaesth 1998; 80: 255-6.

8 Nakata Y, Goto T, Morita S. Comparison of inhalation inductions with xenon and sevoflurane. Acta

Anaesthesiol Scand 1997; 41: 1157-61.

9 Goto T, Saito H, Shinkai M, Nakata $\Upsilon$, Ichinose F, Morita $S$. Xenon provides faster emergence from anesthesia than does nitrous oxide-sevoflurane or nitrous oxide-isoflurane. Anesthesiology 1997; 86: 1273-8.

10 Petersen-Felix S, Luginbühl M, Schnider TW, Curatolo $M$, Arendt-Nielsen L, Zbinden AM. Comparison of the analgesic potency of xenon and nitrous oxide in humans evaluated by experimental pain. Br J Anaesth 1998; 81: 742-7.

11 Stowe DF, Rehmert GC, Kwok W-M, Weigt HU, Georgieff $M$, Bosnjak ZJ. Xenon does not alter cardiac function or major cation currents in isolated guinea pig hearts or myocytes. Anesthesiology 2000; 92: 516-22.

12 Hüneke R, Jüngling E, Skasa M, Rossaint R, Lückhoff $A$. Effects of the anesthetic gases xenon, halothane, and isoflurane on calcium and potassium currents in human atrial cardiomyocytes. Anesthesiology 2001; 95: 999-1006.

13 Luttropp HH, Romner B, Perhag L, Eskilsson J, Fredriksen $S$, Werner $O$. Left ventricular performance and cerebral haemodynamics during xenon anaesthesia. A transoesophageal echocardiography and transcranial Doppler sonography study. Anaesthesia 1993; 48: 1045-9.

14 Morita S, Goto T, Niimi Y, Ichinose F, Saito H. Xenon produces minimal cardiac depression in patients under fentanyl-midazolam anesthesia. Anesthesiology 1996; 85: A362 (abstract).

15 Hettrick DA, Pagel PS, Kersten JR, et al. Cardiovascular effects of xenon in isoflurane-anes- thetized dogs with dilated cardiomyopathy.

Anesthesiology 1998; 89: 1166-73.

16 Yagi M, Mashimo T, Kawaguchi T, Yoshiya I. Analgesic and hypnotic effects of subanaesthetic concentrations of xenon in human volunteers: comparison with nitrous oxide. Br J Anaesth 1995; 74: 670-3.

17 Nakata $\Upsilon$, Goto T, Morita S. Effects of xenon on hemodynamic responses to skin incision in humans. Anesthesiology 1999; 90: 406-10.

18 Marx T, Froeba G, Wagner D, Baeder S, Goertz A, Georgieff $M$. Effects on haemodynamics and catecholamine release of xenon anaesthesia compared with total i.v. anaesthesia in the pig. Br J Anaesth 1997; 78 : 326-7.

19 Goto T, Nakata $\Upsilon$, Ishiguro $\Upsilon$, Niimi $\Upsilon$, Suwa K, Morita $S$. Minimum alveolar concentration-awake of xenon alone and in combination with isoflurane or sevoflurane. Anesthesiology 2000; 93: 1188-93.

20 Goto T, Nakata $\Upsilon$, Saito $H$, Ishiguro $\Upsilon$, Niimi $\Upsilon$, Morita $S$. The midlatency auditory evoked potentials predict responsiveness to verbal commands in patients emerging from anesthesia with xenon, isoflurane, and sevoflurane but not with nitrous oxide. Anesthesiology 2001; 94: 782-9.

21 de Sousa SLM, Dickinson R, Lieb WR, Franks NP. Contrasting synaptic actions of the inhalational general anesthetics isoflurane and xenon. Anesthesiology 2000; 92: 1055-66.

22 Yamakura $T$, Harris $R A$. Effects of gaseous anesthetics nitrous oxide and xenon on ligand-gated ion channels. Comparison with isoflurane and ethanol. Anesthesiology 2000; 93: 1095-101.

23 Marx T, Schmidt M, Schirmer U, Reinelt H. Pollution of the environment and the workplace with anesthetic gases. In: Goto T (Ed.). Xenon. Int Anesthesiol Clin. Lippincott Williams \& Wilkins, 2001; 39: 15-27.

24 Climate Change: Climate Change 1995 - The Science of Climate Change. Cambridge, U.K., The Press Syndicate of the University of Cambridge, 1996. 